J. DIFFERENTIAL GEOMETRY

84 (2010) 19-44

\title{
CONFORMAL BOUNDARY EXTENSIONS OF LORENTZIAN MANIFOLDS
}

\author{
Piotr T. Chruściel
}

\begin{abstract}
We study the question of local and global uniqueness of completions, based on null geodesics, of Lorentzian manifolds. We show local uniqueness of such boundary extensions. We give a necessary and sufficient condition for existence of unique maximal completions. The condition is verified in several situations of interest. This leads to existence and uniqueness of maximal spacelike conformal boundaries, of maximal strongly causal boundaries, as well as uniqueness of conformal boundary extensions for asymptotically simple space-times. Examples of applications include the definition of mass, or the classification of inequivalent extensions across a Cauchy horizon of the Taub space-time.
\end{abstract}

\section{Introduction}

In general relativity one often faces the need of extending the spacetimes under consideration. For example, one might wish to extend a globally hyperbolic space-time by adding a Cauchy horizon [19]. Or one might wish to extend a domain of outer communication, given in some coordinate system, by adding an event horizon $[18,27,28]$. Finally, when studying the asymptotic behavior of the fields, one might wish to add to the space-time a conformal boundary at infinity [23]. In all those cases one makes a "conformal boundary extension" of the original Lorentzian manifold (in the first two cases the conformal factor being one).

It is then natural to raise the question of uniqueness of the extensions. It is the object of this paper to establish some results concerning this problem. We start by proving uniqueness of differentiable structure of the boundary extensions; this is a purely local result. We then pass to an analysis of the global aspects of the problem. We give a necessary and sufficient condition for existence of unique maximal completions in terms of families of null geodesics. We check that our condition is verified in several situations of interest. This leads to a proof of existence and uniqueness of maximal spacelike conformal boundaries. We also obtain uniqueness of conformal boundary extensions for asymptotically simple space-times.

Received 11/2/07. 
The reader is referred to $[4,8,10,11,24-26]$, and also to [15] and references therein, for previous results related to the problems at hand.

This paper is the result of a collaboration with Robert Geroch, who contributed several key ideas and drafted parts of the text.

\section{Preliminaries}

Let $M$ be a smooth $n$-manifold (without boundary), and $g_{a b}$ a smooth, Lorentz-signature metric thereon. (Manifolds are assumed to be paracompact and Hausdorff throughout.)

We will use the convention that a manifold with boundary $\tilde{M}$ contains its boundary $\partial \tilde{M}$ as a point set (recall that it is sometimes convenient, for PDE considerations, not to do that). With this convention, spaces of functions such as, e.g., $C^{k}(\tilde{M})$ consist of functions which are $k$ times continuously differentiable in the interior of $\tilde{M}$, with the derivatives extending by continuity to continuous functions on $\tilde{M}$.

Let $\mathscr{N}_{p} M \subset T_{p} M$ denote the collection of null vectors at $p$, and let $\mathscr{N} M \subset T M$ be the bundle of all null vectors.

Denote by $B \subset T M$ the collection of (nonzero) null vectors $\mu \in \mathscr{N} M$ such that $\exp (\mu)$ exists. Then $B$ is a smooth $(2 n-1)$-manifold (arising as a smooth submanifold of the tangent bundle). Also, the exponential map exp, when restricted to $B$, is a smooth map from $B$ to $M$.

We restrict attention to $(M, g)$ 's which are time oriented, and to those null geodesics which are future directed.

We shall say that a Jacobi field is null-connecting if it arises from a one-parameter differentiable family of null geodesics. (This should not be confused with the usual "quotient modulo the field of tangents" for Jacobi fields along null geodesics; no such quotient will be taken here.)

Consider, then, a differentiable one-parameter family $\gamma(\lambda, s)$ of null geodesics, so that for every $\lambda$ the map $\gamma(\lambda, \cdot)$ is an affinely parameterised null geodesic, set $l=\gamma_{*} \partial_{s}, X=\gamma_{*} \partial_{\lambda}$. It is well known (and in any case easily follows from the vanishing of the torsion) that $\nabla_{X} l=\nabla_{l} X$. Since $g(l, l)=0$ for all geodesics in the family under consideration, one finds

$$
0=X(g(l, l))=2 g\left(l, \nabla_{X} l\right)=2 g\left(l, \nabla_{l} X\right)=2 l(g(l, X)) .
$$

It follows that for all null-connecting Jacobi fields $X$ along a null geodesic $\gamma(s)$ we have

$$
\forall s_{1}, s_{2} \quad g\left(l\left(s_{1}\right), X\left(s_{1}\right)\right)=g\left(l\left(s_{2}\right), X\left(s_{2}\right)\right) .
$$

We shall say that $y$ is null-conjugate to $x$ if $y=\exp \left(s_{0} \mu\right)$ with $s_{0}>0$ for some null vector $\mu \in T_{x} M$ and if there exists a null-connecting Jacobi field along the geodesic segment $\left[0, s_{0}\right] \ni s \rightarrow \exp (s \mu)$ which vanishes precisely at $x$ and at $y$. Note that a null-conjugate point is necessarily a conjugate point in the usual sense, but the inverse implication is false in general. 
From (1) we immediately obtain:

Proposition 2.1. Let $X$ be a null-connecting Jacobi field on a geodesic segment $\gamma$ with $X(0)$ - timelike. Then $X$ has no zeros on $\gamma$.

Proof. Since $X(0)$ is timelike we have $g(\dot{\gamma}(0), X(0)) \neq 0$, and the result follows from (1). q.e.d.

Next, let $\kappa$ be a smooth timelike curve in $M$; note that $\dot{\kappa}$ has no zeros. Denote by $S_{\kappa} \subset B$ the collection of those vectors $\mu \in \mathscr{N} M$ such that i) $\pi(\mu) \in \kappa$ (by an abuse of notation, we will use the same symbol $\kappa$ for a curve and for its image), where $\pi: \mathscr{N} M \rightarrow M$ is the projection map, and ii) the null geodesic segment

$$
t \rightarrow \Gamma_{\mu}(t):=\exp (t \mu), \quad t \in[0,1],
$$

generated by $\mu$ has no null-conjugate points. Thus, $S_{\kappa}$ is an open subset of the $n$-submanifold $\pi^{-1}(\kappa) \subset B$ of $B$, hence a smooth $n$-submanifold of $B$.

Any (non-trivial) flat Riemannian cone (without its tip) multiplied by $(n-2)$-dimensional Minkowski space-time provides an example of space-time in which $\left.\exp \right|_{S_{\kappa}}$ might fail to be injective for some timelike curves $\kappa$. We note that there are no null-conjugate points in this spacetime.

Proposition 2.2. The map

$$
S_{\kappa} \stackrel{\exp }{\rightarrow} \exp \left(S_{\kappa}\right) \subset M
$$

is a submersion.

Proof. The usual analysis of Jacobi fields shows that $\operatorname{Ker}\left(\left(\left.\exp \right|_{S_{\kappa}}\right)_{*}\right) \neq\{0\}$ if and only if there exist null-connecting Jacobi fields along $\Gamma_{\mu}$ which vanish at $\exp (\mu)$ and which are initially tangent to $\kappa$, possibly vanishing there. If $X(0)$ vanishes, then the fact that $X$ has no other zeros follows from the definition of $S_{\kappa}$. If $X(0) \neq 0$, then $X(0)$ is timelike since it is tangent to $\kappa$, and the fact that $X$ is nowhere vanishing follows from Proposition 2.1. $\quad$ q.e.d.

Let $S_{\kappa}^{\prime}$ denote any open subset of $S_{\kappa}$ with the property that $\left.\exp \right|_{S_{\kappa}^{\prime}}$ is injective. It then follows from Proposition 2.2 that $\left.\exp \right|_{S_{\kappa}^{\prime}}$ is a diffeomorphism into its image.

Choose any such $S_{\kappa}^{\prime}$ and denote by $f$ the function on the $n$-manifold $S_{\kappa}^{\prime}$ given by " $\kappa$-parameter value": if $s$ is a parameter along $\kappa$, then

$$
f(\kappa(s), \mu):=s \text {; }
$$

so $f$ is smooth and has nowhere vanishing gradient. Its composition with $\left(\left.\exp \right|_{S_{\kappa}^{\prime}}\right)^{-1}$ (resulting in a function on $S_{\kappa}^{\prime}$ ), then, is also smooth on its domain of definition and has nowhere vanishing gradient there. In fact, we have the following: 
Proposition 2.3. The gradient of

$$
u_{\kappa}:=f \circ \exp ^{-1}
$$

at $\exp (\mu) \in \exp \left(S_{\kappa}^{\prime}\right) \subset M$ is proportional to the tangent, at this point, to the geodesic generated by $\mu$, with non-zero proportionality factor.

REMARK 2.4. Some comments about differentiability thresholds are in order: our analysis requires local uniqueness of geodesics, as well as controlled behavior of Jacobi fields. Those properties will hold if the metric is $C^{k, 1}$, with $k \geq 1$. Such differentiability of the metric leads naturally to $C^{k+1,1}$ differentiability of the manifold. The exponential map is then a $C^{k-1,1}$ map (both as a function of $\mu \in T_{\pi(\mu)} M$ and of $\pi(\mu)$ ), while Jacobi fields are of $C^{k-2,1}$ differentiability class (since the Jacobi equation involves the Riemann tensor) as functions of initial data at $p \in M$ and of $p$. Because Jacobi fields govern the differentiability of families of geodesics, the field of tangents to the level sets of $u$ will be $C^{k-2,1}$ differentiability class. This results in $C^{k-1,1}$ differentiability of $u_{\kappa}$ when $\kappa$ is $C^{k-1,1}$ or better. This is a loss of two derivatives as compared to the expected differentiability class of $M$.

REMARK 2.5. We also note that, for solutions of field equations, a possible hypothesis on the metric near a conformal boundary at infinity $\grave{a}$ la Penrose is that of polyhomogeneity; see, e.g., [7,17]. If $\kappa$ is smooth, then $u_{k}$ will be polyhomogeneous and $C^{k+1}$ at the conformal boundary whenever the metric is polyhomogeneous and $C^{k}$ there, $k \geq 1$. (The increase of differentiability, as compared to Remark 2.4, arises from the fact that integrals, along curves which are transverse to the boundary, of polyhomogeneous functions increase differentiability by one; this gives $C^{k}$ and polyhomogeneous Jacobi fields, hence a $C^{k}$ field of null normals at the conformal boundary, hence a $C^{k+1}$ and polyhomogeneous function $u_{\kappa}$ by the analysis of [7, Appendix B].)

Proof. The function $f \circ \exp ^{-1}$ is constant along the future light cones issued from $x(s) \in \kappa$, hence its gradient is proportional to the normal to those light cones. Since the subsets of those light cones which lie in the image by exp of $S_{\kappa}^{\prime}$ form smooth null hypersurfaces, their normals are proportional to the tangents of their geodesic generators. The fact that the proportionality factor is non-zero follows from the fact that $\left.\exp \right|_{S_{\kappa}^{\prime}}$ is a diffeomorphism into its image and that $f$ has non-vanishing gradient on $S_{\kappa}^{\prime}$.

q.e.d.

The functions $u_{\kappa}$ as in (4) provide the main tool for our local analysis below.

Next, let $L$ be a smooth $(n-1)$-submanifold of $M$. We will actually be mainly interested in situations when $L$ is a boundary of $M$; in order to make this compatible with our hypotheses above, we then extend $M$ to a new manifold, still denoted by $M$. Thus, without loss of generality, 
we can assume that $L$ is an interior submanifold of $M$; the discussion above is independent of the extensions done. Denote by $B_{L} \subset B$ those points $\mu$ such that i) $\exp \mu \in L$, and ii) the null geodesic generated by $\mu$ meets $L$ transversally (with all intersections transverse, whenever there is more than one). Then $B_{L}$ is a smooth $(2 n-2)$-(sub)manifold (of $B)$. Fix $\mu \in B_{L}$. Denote by $\Gamma$ the tangent, at $\mu$, to the curve in $B$ given by $\exp (a \mu)$, with $a$ in the domain of definition of $\exp (\cdot)$. Then, by transversality, $\Gamma$ is not tangent to the submanifold $B_{L}$. That is, this curve meets $B_{L}$, at $a=1$, transversally. So, since $B_{L}$ has codimension one in $B$, all nearby such curves (i.e., $\exp \left(a \mu^{\prime}\right)$, for $\mu^{\prime}$ near $\mu$ ) also meet $B_{L}$, and also transversally. In other words, all null geodesics generated by elements near $\mu$ meet the original submanifold $L$, also transversally.

\section{Local uniqueness of conformal boundary extensions}

The following result establishes uniqueness of the differentiable structure of conformal boundary extensions under suitable hypotheses:

Theorem 3.1. Let $M$ be a smooth n-manifold, $n \geq 3$, and $g_{a b} a$ smooth Lorentz-signature metric thereon. Let $\left(\hat{M}, \hat{g}_{a b}\right)$ be a smooth $n$ manifold with boundary with smooth Lorentz-signature metric, and let $M \stackrel{\psi}{\rightarrow} \hat{M}$ be a smooth diffeomorphism into, such that i) $\psi[M]=\hat{M} \backslash \partial \hat{M}$, and $i i)$ the $\psi$-image of $g_{a b}$ is conformal to $\hat{g}_{a b}$. Similarly for $\hat{M}^{\prime}, \hat{g}_{a b}^{\prime}$, and $\psi^{\prime}$. Let $I \stackrel{\gamma}{\rightarrow} M$, where $I$ is an open interval in $\mathbb{R}$, be a directed null geodesic in $\left(M, g_{a b}\right)$ such that $\hat{\gamma}=\psi \circ \gamma$ has future endpoint $p \in \hat{M}$ and $\hat{\gamma}^{\prime}=\psi^{\prime} \circ \gamma$ has future endpoint $p^{\prime} \in \hat{M}^{\prime}$. We also assume that $\hat{\gamma}$ meets $\partial \hat{M}$, and $\hat{\gamma}^{\prime}$ meets $\partial\left[\hat{M}^{\prime}\right]$, transversally. Then there exist open neighborhoods $U$ of $p$ in $\hat{M}$ and $U^{\prime}$ of $p^{\prime}$ in $\hat{M}^{\prime}$, together with a smooth diffeomorphism $U \stackrel{\phi}{\rightarrow} U^{\prime}$, such that I) $\phi(p)=p^{\prime}$, and II) restricted to $U \cap \psi[M], \psi^{\prime} \circ \psi^{-1}=\phi$.

REMARK 3.2. The result is wrong in space-time dimension two. In order to see that, let $M$ be the 2 -manifold with chart $(u, v)$ where $u<0$, $v \in \mathbb{R}$. Let $g$ be the metric $d u d v$ on $M$. Let $\gamma$ be the null geodesic given by $u=\lambda, v=0$, where $\lambda$ (the parameter) goes from $-\infty$ to 0 . We introduce two conformal boundary completions in which $\gamma$ acquires an endpoint.

We let, first, $M^{\prime}$ be the obvious completion: thus $M^{\prime}$ is the manifold with boundary with chart $(a, b)$, where $a \leq 0, b \in \mathbb{R}$; with metric $d a d b$. Let $\psi^{\prime}$ be the mapping from $M$ to $M^{\prime}$ that sends $(u, v)$ to the point $a=u, b=v$. This is a conformal boundary extension (with conformal factor one) terminating $\gamma$; by this we mean that $\gamma$ has an endpoint at the boundary.

We let, next, $M^{\prime \prime}$ be the manifold with boundary with chart $(p, q)$, where $p \leq 0, q \in \mathbb{R}$; with metric $d p d q$. Let $\psi^{\prime \prime}$ be the mapping from 
$M$ to $M^{\prime \prime}$ that sends $(u, v)$ to the point $p=-\sqrt{-u}, q=v$. This is again a conformal boundary extension (with non-trivial conformal factor), terminating $\gamma$.

Clearly the map $\psi^{\prime \prime} \circ\left(\psi^{\prime}\right)^{-1}$ does not satisfy the conclusions of Theorem 3.1 .

REMARK 3.3. The main situations of interest in general relativity are with a conformal factor identically one, or with a conformal factor relating $g$ and $\hat{g}$ that degenerates at $\partial \hat{M}$ to first order; similarly for $\hat{g}^{\prime}$. We emphasise that no such restrictions are made here. (On the other hand, we expect that in dimension two the proof of Theorem 3.1 can be adapted with such supplementary assumptions.)

Remark 3.4. If $C^{k+1,1}$ differentiability of the manifolds and $C^{k, 1}$ differentiability of the metrics involved is assumed, $k \geq 1$, then the result remains true with $\phi$ of $C^{k-1,1}$ differentiability class (see Remark 2.4). On the other hand, if the metric is assumed to be polyhomogeneous and $C^{k}, k \geq 1$, near the conformal boundary, then $\phi$ will be polyhomogeneous and $C^{k+1}$ (see Remark 2.5).

Proof. Let $q$ be a point of $\hat{\gamma}, \lambda$ the direction of the curve $\hat{\gamma}$ at $q, A$ an open neighborhood of $\lambda$ in the $(2 n-2)$-manifold of null directions at points of $\hat{M} \backslash \partial \hat{M}$, and $U$ the (open) subset of $\hat{M}$ consisting of the union of null rays generated by all points of $A$. Then, we claim,

Proposition 3.5. For all $q$ sufficiently close to $p$, and all $A$ sufficiently small, there exists at most one $\hat{g}$-geodesic between any two points in $U$, and:

1) The spacetime $U$ is strongly causal,

2) every null ray generated by a point of $A$ has future endpoint on $\partial \hat{M}$ and meets $\partial \hat{M}$ transversally there, and

3) no null geodesic segment contained in $U$ has a pair of conjugate points in $\left(U,\left.\hat{g}\right|_{U}\right)$.

Proof. Let $O$ be a geodesically convex neighborhood of $p$.

1) We take a strongly causal neighborhood of the point $p$ which is included in $O$. Given any $q$ such that the segment $[q, p]$ of $\hat{\gamma}$ lies entirely within that neighborhood and any $A$ sufficiently small we will have that $U$ lies entirely within that neighborhood.

2) This follows from transversality of $\hat{\gamma}$ at $\hat{p}$ and from continuous dependence of solutions of ODE's upon initial values.

3) Let $\hat{h}$ be any auxiliary Riemannian metric on $\hat{M}$; the result follows from the fact that the uniform bound on the Riemann tensor of $\hat{g}$ on a compact set allows one to control from below the $\hat{h}$-distance to a conjugate point. 
Let $\hat{q}=\psi(q)$ be close enough to $p$ so that the conclusions of Proposition 3.5 hold for some open neighborhood $\hat{A}=\psi_{*}(A)$ of $\hat{\lambda}=\psi_{*} \lambda \in T_{\hat{q}} \hat{M}$, as determined by the boundary $\partial \hat{M}$ in the space-time $(\hat{M}, \hat{g})$, and that the same conclusions apply to some open neighborhood $\hat{A}^{\prime}=\psi_{*}^{\prime}\left(A^{\prime}\right)$ as determined by the boundary $\partial \hat{M}^{\prime}$ in the space-time $\left(\hat{M}^{\prime}, \hat{g}^{\prime}\right)$. Replacing $A$ by $A \cap A^{\prime}$, one then finds that the conclusions of Proposition 3.5 simultaneously hold for $\psi_{*}(A)$ and for $\psi_{*}^{\prime}(A)$, with sets $\hat{U} \subset \hat{M}$ and $\hat{U}^{\prime} \subset \hat{M}^{\prime}$.

We let $\pi$ denote the projection from $T M$ to $M$.

Replacing $\hat{M}$ by $U$, we can without loss of generality assume that $\hat{M}$ is strongly causal. This implies that, close enough to $p \in \hat{M}$, the boundary $\dot{J}^{-}(p)$ of the causal past of $p$, after removal of $p$, is a smooth submanifold of $\hat{M}$.

We wish to construct convenient coordinate systems near the boundary, the following assertion will be useful:

Proposition 3.6. Let $n \geq 3$ and let $\mathscr{U}$ be a neighborhood of $p \in \partial \hat{M}$ such that $\left(\dot{J}^{-}(p) \cap \mathscr{U}\right) \backslash\{p\}$ is a smooth submanifold. Denote by $\tilde{K}$ the collection of all null vectors $l$, with $\pi(l) \in \psi^{-1}\left(\dot{J}^{-}(p) \cap \mathscr{U}\right) \subset M$, such that the corresponding null geodesic in $\psi(M)=\hat{M} \backslash \partial \hat{M}$ ends at $p \in \partial \hat{M}$, and there its tangent is transverse to the boundary of $\hat{M}$. Let $K$ denote the connected component of $\tilde{K}$ containing all the null vectors $\dot{\gamma}$ (where $\gamma$ is as in the statement of Theorem 3.1), and let $W$ be the subset of

$$
\underbrace{K \times \ldots \times K}_{n \text { factors }}
$$

consisting of $n$-tuples such that the null-tangents to the corresponding null geodesics are linearly independent at $p$. Then $W$ is open and dense in $K \times \ldots \times K$.

Proof. Let $\alpha: K \rightarrow T_{p} \hat{M}$ denote the map which to a vector $l \in$ $K \subset T_{q} M$ assigns the tangent at $p$ to the corresponding affinely parameterised null geodesic passing through $q$, with tangent $l$ there. Then $\alpha$ is continuous on $K$. Let $\left(\alpha\left(l_{1}\right) \ldots \alpha\left(l_{n}\right)\right)$ denote the matrix obtained by juxtaposition of the vectors $\alpha\left(l_{i}\right)$, then $W=\left\{\operatorname{det}\left(\alpha\left(l_{1}\right) \ldots \alpha\left(l_{n}\right)\right) \neq 0\right\}$, hence $W$ is open.

Consider any point $\left(l_{1}, \ldots, l_{n}\right)$ in $K \backslash W$, and let $k<n$ be the rank of $\left(\alpha\left(l_{1}\right) \ldots \alpha\left(l_{n}\right)\right)$. By reordering we can without loss of generality assume that $\left\{\alpha\left(l_{1}\right), \ldots, \alpha\left(l_{k}\right)\right\}$ are linearly independent. Suppose that there exists an open set $\mathscr{O} \subset M$ around $q_{k+1}=\pi\left(l_{k+1}\right)$ such that $\alpha(x) \in \operatorname{Vect}\left\{\alpha\left(l_{1}\right), \ldots, \alpha\left(l_{k}\right)\right\}$ for all $l \in K \cap \pi^{-1}(\mathscr{O})$, then $\pi(K) \cap \mathscr{O}$ is a subset of the smooth $(k-1)$-dimensional manifold

$$
\psi^{-1}\left(\left\{\exp (m), m \in \operatorname{Vect}\left\{\alpha\left(l_{1}\right), \ldots, \alpha\left(l_{k}\right)\right\}, g(m, m)=0\right\} \cap \mathscr{U}\right) .
$$


However, $\pi(K) \cap \mathscr{O}=\dot{J}^{-}(p) \cap \mathscr{O}$; since $\dot{J}^{-}(p)$ is an achronal boundary, the set $\dot{J}^{-}(p) \cap \mathscr{O}$ is an $(n-1)$-dimensional topological submanifold, which gives a contradiction. It follows that there exists an $\epsilon$ perturbation of $l_{k+1}$ such that the rank of $\left(\alpha\left(l_{1}\right) \ldots \alpha\left(l_{n}\right)\right)$ at the perturbed points $l_{i}$ is $k+1$. In a finite number of steps one obtains a perturbation, as small as desired, such that the rank of $\left(\alpha\left(l_{1}\right) \ldots \alpha\left(l_{n}\right)\right)$ at the perturbed points $l_{i}$ is $n$.

q.e.d.

Let $\mathscr{U}^{\prime}$ be a set in $\hat{M}^{\prime}$ as in Proposition 3.6, passing to subsets we can assume that $\psi^{\prime-1}\left(\mathscr{U}^{\prime}\right)=\psi^{-1}(\mathscr{U})$. Let $K^{\prime}$ be the analogous collection as determined by $p^{\prime}$ and $\hat{M}^{\prime}$. It is not completely clear whether or not $K=K^{\prime}$; however, $K \cap K^{\prime}$ forms a neighborhood, both within $K$ and $K^{\prime}$, of all the points $\dot{\gamma}(s)$. Let $W^{\prime}$ be the corresponding $W$ set; then $W \cap W^{\prime}$ is still open and dense in $\left(K \cap K^{\prime}\right) \times \ldots \times\left(K \cap K^{\prime}\right)$. Further, the null-tangents to the corresponding null geodesics are now linearly independent both at $p \in \partial \hat{M}$ and at $p^{\prime} \in \partial \hat{M}^{\prime}$.

Let

$$
(\ell(1), \ldots, \ell(n)) \in W \cap W^{\prime} \cap(A \times \ldots \times A) ;
$$

this last set is not empty in view of what has been said. Choose $n$ smooth, timelike curves $\kappa(a), a=1, \ldots, n$, in $M$ such that $\kappa(a)$ passes through $q(a)$. The construction before Proposition 2.3 applies to $\left(\hat{U},\left.\hat{g}\right|_{\hat{U}}\right)$ and leads to $n$ smooth functions $u_{\psi \circ \kappa(a)}$, associated with the timelike curves $\psi \circ \kappa(a)$, defined throughout $\hat{U}$. One can likewise carry that construction on $\left(\hat{U}^{\prime},\left.\hat{g}^{\prime}\right|_{\hat{U}^{\prime}}\right)$, using the curves $\psi^{\prime} \circ \kappa(a)$, obtaining $n$ functions $u_{\psi^{\prime} \circ \kappa(a)}$. Clearly

$$
u_{\psi \circ \kappa(a)} \circ \psi=u_{\psi^{\prime} \circ \kappa(a)} \circ \psi^{\prime} .
$$

By construction the gradients of the $u_{\psi \circ \kappa(a)}$ 's are linearly independent at $p$; similarly the gradients of the $u_{\psi^{\prime} \circ \kappa(a)}$ 's are linearly independent at $p^{\prime}$. Passing to subsets of $\hat{U}$ and $\hat{U}^{\prime}$ if necessary, by continuity those gradients will be linearly independent throughout $\hat{U}$ and $\hat{U}^{\prime}$. It follows that $\left\{u_{\psi \circ \kappa(a)}\right\}_{a=1, \ldots, n}$ forms an $\hat{M}$-admissible coordinate system in a neighborhood of $p$; similarly for $\left\{u_{\psi^{\prime} \circ \kappa(a)}\right\}_{a=1, \ldots, n}$. The map $\phi$ is defined to be the identity in the coordinate systems just constructed. It should be clear that I) holds, while II) is equivalent to (5).

\section{Maximal extensions}

Suppose that a manifold without boundary $(M, g)$ has been conformally extended to a manifold with boundary $(\hat{M}, \hat{g})$. One can then always produce a smaller extension by removing points from $\partial \hat{M}$. This is a rather trivial operation, which can be reverted by adding back the points that have been discarded. One is thus led to the notion of maximal extension: this is an extension which cannot be "made larger by 
adding points." Then the question arises, how many maximal extensions exist? This is the issue that we want to address in this section. We start by developing some terminology, and tools, which will be used to handle the problems at hand.

Fix, once and for all, a smooth $n$-dimensional manifold $M$ (without boundary), and a smooth, Lorentz-signature metric, $g$, thereon. By a conformal boundary extension of a pseudo-Riemannian manifold $(M, g)$, we mean a triple, $\alpha^{\prime}=\left(M^{\prime}, g^{\prime}, \psi^{\prime}\right)$, where $M^{\prime}$ is a smooth manifold (possibly) with boundary, $g^{\prime}$ is a smooth, Lorentz-signature metric on $M^{\prime}$, and $\psi^{\prime}$ is a smooth mapping, $M \stackrel{\psi^{\prime}}{\rightarrow} M^{\prime}$, such that $\psi^{\prime}$ i) is a diffeomorphism into, ii) satisfies $\psi^{\prime}[M]=M^{\prime}-\partial M^{\prime}$, with iii) $g$ being conformal to $\left(\psi^{\prime}\right)^{*} g^{\prime}$.

We note that the question of the behavior of the relative conformal factor when the boundary is approached is completely irrelevant from our point of view, so our analysis covers the usual Penrose-type situations where the inverse of the conformal factor vanishes throughout the boundary (we will talk about conformal completions at infinity in this case), but also situations where the conformal factor equals one, but also situations where the conformal factor tends to any values in $\mathbb{R}^{+} \cup\{\infty\}$ as one approaches the boundary, or perhaps does not even have limits at the boundary points.

When we write $\alpha^{\prime \prime}$, the associated objects will be implicitly understood as $\left(M^{\prime \prime}, g^{\prime \prime}, \psi^{\prime \prime}\right)$, similarly for $\alpha_{a}$, $\hat{\alpha}$, etc.

A conformal boundary extension $\alpha^{\prime}$ will be called future if every point of $\partial M^{\prime}$ is the future endpoint of some null geodesic; similarly one can talk about past conformal boundary extensions. It is easily seen that every point in a future extension terminates some null geodesic transversally. Note that a future conformal boundary extension can simultaneously be a past one.

From now on we restrict attention to future extensions. The analysis below applies to past extensions, or to extensions, after obvious modifications, using past inextendible, or inextendible, null geodesics. Note, however, that there are situations where the constructions below using simultaneously future and past extensions yield an empty maximal boundary, while non-trivial future and past boundaries exist.

Given a manifold with boundary $M$, we use the symbol $M$ to denote its interior, $\stackrel{\circ}{M}:=M \backslash \partial M$.

Given any two conformal boundary extensions $\alpha^{\prime}$ and $\alpha^{\prime \prime}$ we write $\alpha^{\prime} \leq \alpha^{\prime \prime}$ provided there exists a smooth conformal diffeomorphism into $M^{\prime} \stackrel{\phi}{\rightarrow} M^{\prime \prime}$ satisfying $\phi \circ \psi^{\prime}=\psi^{\prime \prime}$. We write $\alpha^{\prime} \leq_{\phi} \alpha^{\prime \prime}$ when the map $\phi$ has been chosen.

We write $\alpha^{\prime} \sim \alpha^{\prime \prime}$ if there exists a $\phi$ as above which is a diffeomorphism of manifolds with boundary. This is obviously an equivalence relation. 
The reader should note that two equivalent extensions are conformal to each other, but sometimes extensions which are conformal to each other might fail to be equivalent. The following simple example illustrates this: Let $M$ have coordinates $(u, v)$ each with range $(-1,1)$, with metric $d u d v$. Let $M^{\prime}$ have global coordinates

$$
\left(u^{\prime}, v^{\prime}\right) \in(-1,1] \times(-1,1] \backslash\{(1,1)\},
$$

with metric $d u^{\prime} d v^{\prime}$. Let $\psi^{\prime}$ send $(u, v)$ in $M$ to $u^{\prime}=u, v^{\prime}=v$ in $M^{\prime}$. Let $M^{\prime \prime}$ have global coordinates

$$
\left(u^{\prime}, v^{\prime}\right) \in[-1,1) \times[-1,1) \backslash\{(-1,-1)\},
$$

with metric $d u^{\prime \prime} d v^{\prime \prime}$. Let, finally, $\psi^{\prime \prime}$ send $(u, v)$ in $M$ to $u^{\prime \prime}=u, v^{\prime \prime}=v$ in $M^{\prime \prime}$. So, we now have a space-time, and two conformal boundary extensions $\alpha^{\prime}$ and $\alpha^{\prime \prime}$. Clearly $\left(M, g^{\prime}\right)$ is isometric with $\left(M^{\prime \prime}, g^{\prime \prime}\right)$, the relevant isometry being the map which sends $\left(u^{\prime}, v^{\prime}\right)$ to $\left(u^{\prime \prime}, v^{\prime \prime}\right)=$ $\left(-u^{\prime},-v^{\prime}\right)$. However, $\alpha^{\prime} \nsim \alpha^{\prime \prime}$. Indeed, suppose that there exists $\psi$ such that $\psi \circ \psi^{\prime}=\psi^{\prime \prime}$, then for $(u, v) \in \dot{M}^{\prime}$ we have

$$
\psi(u, v)=\psi \circ \psi^{\prime}(u, v)=\psi^{\prime \prime}(u, v)=(u, v),
$$

so that $\psi(u, v)=(u, v)$, which does not extend to a map from $\partial M^{\prime}$ to $M^{\prime \prime}$.

A less trivial example of the above behavior is given by the two standard extensions of the Taub region of Taub-NUT space-time, which again are isometric but nonequivalent.

In our new terminology, Theorem 3.1 is equivalent to the following statement:

Theorem 4.1. Let $(M, g)$ be a spacetime, $\gamma$ a future-inextendible null geodesic therein, and $\alpha$ and $\alpha^{\prime}$ conformal extensions in each of which $\gamma$ acquires a transversal future endpoint. Then there exists a conformal extension, $\alpha^{\prime \prime}$, which is smaller than both $\alpha$ and $\alpha^{\prime}$, and in which $\gamma$ also acquires a transversal future endpoint.

It follows immediately from the definitions above that if $\alpha^{\prime} \leq_{\phi_{1}} \alpha^{\prime \prime}$ and $\alpha^{\prime \prime} \leq_{\phi_{2}} \alpha^{\prime}$, then $\phi_{1}$ and $\phi_{2}$ are inverses of each other on the interiors $\dot{M}^{\prime}$ and $\dot{M}^{\prime \prime}$ :

$$
\left.\phi_{1} \circ \phi_{2}\right|_{\stackrel{\circ}{M}^{\prime}}=\operatorname{Id}_{\dot{M}^{\prime}},\left.\quad \phi_{2} \circ \phi_{1}\right|_{\stackrel{\circ}{M}^{\prime \prime}}=\operatorname{Id}_{M^{\circ}} .
$$

Because both $\phi_{1}$ and $\phi_{2}$ smoothly extend by continuity to the boundaries of their domains of definition by hypothesis, we claim that

$$
\phi_{1} \circ \phi_{2}=\operatorname{Id}_{M^{\prime}}, \quad \phi_{2} \circ \phi_{1}=\operatorname{Id}_{M^{\prime \prime}} .
$$

Indeed, consider a sequence $p_{n} \in \stackrel{\circ}{M}^{\prime}$ such that $p_{n} \rightarrow p \in \partial M^{\prime}$, then $\phi_{2}\left(p_{n}\right)$ converges to $\phi_{2}(p)$, so that the left-hand side of (6) converges to $\phi_{1}\left(\phi_{2}(p)\right)$, while the right-hand side converges to $p$. We have therefore obtained: 
Proposition 4.2. The relation $\leq i$ is a partial ordering on the set of $\sim$-equivalence classes (we will still use the same symbol $\leq$ for that new relation).

We want to construct maximal future conformal boundary extensions. However, the Taub-NUT example shows that completions obtained by "attaching everything that can be attached" might lead to non-Hausdorff behavior. In order to gain insight in the phenomena that occur, some further terminology will be needed:

Definition 4.3. Let $\gamma_{1}$ and $\gamma_{2}$ be null geodesics which are maximally extended to the future.

1) For $x \in M$ and $l$ a null vector at $x$, by a thickening of the corresponding null geodesic we mean the union of all null geodesics arising from initial conditions in a (TM-)neighborhood of $l$.

2) We shall say that the pair $\left(\gamma_{1}, \gamma_{2}\right)$ is simple if either i) each null geodesic enters and remains in every thickening of the other; or if ii) after moving far enough along each null geodesic one can find thickenings $\mathscr{O}_{a}$ of each, $a=1,2$, that have empty intersection, $\mathscr{O}_{1} \cap \mathscr{O}_{2}=\emptyset$.

3) We shall say that $\gamma_{1}$ and $\gamma_{2}$ are intertwined if $\left(\gamma_{1}, \gamma_{2}\right)$ is not simple.

4) A collection $\Omega$ of null future inextendible geodesics will be called simple if $\Omega$ is open and if all pairs $\left(\gamma_{1}, \gamma_{2}\right) \in \Omega \times \Omega$ are simple.

5) A simple collection $\Omega$ of null future inextendible geodesics will be called maximal if for any open set $\Omega_{1}$ of future inextendible null geodesics the collection $\Omega \cup \Omega_{1}$ is not simple.

An equivalent way of saying that two geodesics are intertwined is the following: For any sufficiently small thickening of either, the other null geodesic intersects that thickening in a set that has infinitely many connected components.

The reader will easily check that any collection of future inextendible null geodesics in Minkowski space-time is simple. (This also follows from Theorems 5.9 and 5.10 below.) On the other hand, the collection of all future inextendible null geodesics in Taub-NUT space-time is not, compare Section 5.6.

The set of simple collections of null geodesics is directed by inclusion, and it is a simple consequence of the Kuratowski-Zorn lemma that for every simple $\Omega$ there exists a simple maximal $\hat{\Omega}$ such that $\Omega \subset \hat{\Omega}$. Examples, e.g., in polarized Gowdy space-times with Cauchy horizons (see [5]), show that there might be more than one such $\hat{\Omega}$.

Conformal boundaries provide simple collections of null geodesics:

Proposition 4.4. Let $\hat{\alpha}$ be a conformal boundary extension of $(M, g)$, and let $\Omega(\hat{\alpha})$ denote the collection of maximally extended null geodesics 
in $M$ which acquire a transverse future endpoint on the conformal boundary $\partial \hat{M}$. Then $\Omega(\hat{\alpha})$ is simple.

Proof. Openness of $\Omega(\hat{\alpha})$ is obvious. Let $\gamma_{a}, a=1,2$, be two null geodesics in $\Omega(\hat{\alpha})$, and let $\hat{\gamma}_{a}$ be their future inexentendible extensions in $\hat{M}$. Then the $\hat{\gamma}_{a}$ 's possess endpoints $p_{a}$ on the boundary $\partial \hat{M}$ of $\hat{M}$, with null tangents $m_{a} \in T_{p_{a}} M$ there with respect to some convenient parameterisation; for instance, one can use the distance with respect to some chosen smooth-up-to boundary Riemannian metric on $\hat{M}$. If $p_{1}=p_{2}$ we are in case $i$ ) of Definition 4.3. Otherwise, we can find small disjoint neighborhoods $\mathscr{U}_{a} \subset T \hat{M}$ of $\left(p_{a}, m_{a}\right)$ so that $\exp \left(\mathscr{U}_{1}\right) \cap$ $\exp \left(\mathscr{U}_{2}\right)=\emptyset$. (Here we view exp as a map from $T \hat{M}$ to $\hat{M}$.) Then any thickening of $\gamma_{a}$ contained within $\exp \left(\mathscr{U}_{a}\right) \cap M$ will satisfy the condition of case $i i$ ) of Definition 4.3. $\quad$ q.e.d.

We are ready now to address the question, when does a family of conformal boundary extensions arise from a single larger one?

Theorem 4.5. Let $(M, g)$ be a space-time (without boundary), and consider any non-empty collection $\Xi$ of future conformal boundary extensions. There exists an extension $\alpha \Xi$ that is larger than or equal to every one of these if and only if no two extensions in $\Xi$ terminate intertwined null geodesics.

REMARK 4.6. The reader will notice that $M_{\Xi}$ constructed in the proof below is the minimal one which is larger than all the extensions in $\Xi$, hence unique.

Proof. The necessity of the condition follows from Proposition 4.4, it remains to show sufficiency.

For $\alpha^{\prime}=\left(M^{\prime}, g^{\prime}, \psi^{\prime}\right)$ any conformal boundary extension in $\Xi$, denote by $B_{\alpha^{\prime}}$ the collection of all pairs, $(x, l)$, where $x \in M$, and $l$ is a null vector in $M$ at $x$, such that the affinely parameterized null geodesic in $M^{\prime}$, with initial condition the $\psi^{\prime}$-image of $(x, l)$, meets the boundary of $M^{\prime}$, transversally. Then $B_{\alpha^{\prime}}$ is open in the null bundle of $M$.

Denote by $B_{\Xi}$ the union of the $B_{\alpha^{\prime}}$, over all conformal boundary extensions $\alpha^{\prime} \in \Xi$. Call two points of $B_{\Xi}$ equivalent if there exists a conformal boundary extension in which the two corresponding null geodesics (in the conformal boundary extension manifold) meet at the same boundary point (of that conformal boundary extension manifold). This is an equivalence relation; the only non-trivial property to check is transitivity: Suppose, thus, that there exists a completion $\alpha^{\prime}=\left(M^{\prime}, g^{\prime}, \psi^{\prime}\right)$ in which the maximal extensions of the null geodesics $\psi^{\prime}\left(\gamma_{1}\right)$ and $\psi^{\prime}\left(\gamma_{2}\right)$ meet the boundary $\partial M^{\prime}$ transversally at $p^{\prime}$, and that there exists a completion $\alpha^{\prime \prime}=\left(M^{\prime \prime}, g^{\prime \prime}, \psi^{\prime \prime}\right)$ in which the maximal extensions of the null geodesics $\psi^{\prime \prime}\left(\gamma_{2}\right)$ and $\psi^{\prime \prime}\left(\gamma_{3}\right)$ meet the boundary 
$\partial M^{\prime}$ transversally at $p^{\prime \prime}$. By Theorem 3.1 the map $\psi^{\prime \prime} \circ\left(\psi^{\prime}\right)^{-1}$ extends smoothly to $\partial M^{\prime \prime}$, so that the maximal extension of the geodesic $\psi^{\prime \prime}\left(\gamma_{1}\right)=\psi^{\prime \prime} \circ\left(\psi^{\prime}\right)^{-1}\left(\psi^{\prime}\left(\gamma_{1}\right)\right)$ meets $\partial M^{\prime \prime}$ transversally at $p^{\prime \prime}$, and transitivity follows.

Set $M_{\Xi}$ the disjoint union of $M$ and the set of equivalence classes. Now, given any conformal boundary extension in $\Xi, \alpha^{\prime}=\left(M^{\prime}, g^{\prime}, \psi^{\prime}\right)$, we consider the mapping $M^{\prime} \stackrel{\zeta^{\prime}}{\rightarrow} M_{\Xi}$, given as follows. For $x$ any point of $M$, this map $\zeta^{\prime}$ sends $\psi^{\prime}(x) \in M^{\prime}$ to the point $x \in M$ on the left $(M)$ side of the disjoint union that is $M_{\Xi}$. For $p \in M^{\prime}$ on the boundary, $\zeta^{\prime}$ sends $p$ to the equivalence class of elements of $B_{\Xi}$ consisting of those that generate the geodesics (in $M^{\prime}$ ) that meet at the boundary point $p$ of $M^{\prime}$. The images of these maps $\zeta^{\prime}$ (for the various conformal boundary extensions) cover $M_{\Xi}$ by construction, as any boundary point of a conformal boundary extension is the endpoint of some null transverse geodesic. To see that the $\zeta^{\prime \prime}$ s are injective, let $\zeta^{\prime}\left(p_{1}\right)=\zeta^{\prime}\left(p_{2}\right)$; thus there exists a conformal boundary extension $\alpha^{\prime \prime}$ and null geodesics $\gamma_{1}$ and $\gamma_{2}$ which acquire endpoints $p_{1}$ and $p_{2}$ on $\partial M^{\prime}$, and acquire the same endpoint $p^{\prime \prime}$ on $\partial M^{\prime \prime}$. This last property implies that we are in case $i$ ) of point 2 of Definition 4.3 , and it easily follows that $p_{1}=p_{2}$.

We now introduce charts on the set $M_{\Xi}$ by taking charts on conformal boundary extensions, and sending them to $M_{\Xi}$ via the map $\zeta^{\prime}$. These cover $M_{\Xi}$; any two of them arising from a single completion are obviously compatible, while any two arising from two different completions can be seen to be compatible by Theorem 3.1: Indeed, consider two coordinate charts, one around a boundary point $p^{\prime}$ in a completion $\alpha^{\prime}$, and a second one around a corresponding boundary point $p^{\prime \prime}$ in a completion $\alpha^{\prime \prime}$. In order to apply Theorem 3.1 we need to find a null geodesic which will be transverse to the conformal boundary in both completions. Let $K^{\prime}$ be the set $K$ associated with $p^{\prime}$, as defined in Proposition 3.6. Then $K^{\prime}$ is open and dense in the set of all $(q, \ell)$ in $T M$ such that $\ell$ is null, and such that the associated null geodesic ends at $p^{\prime}$. Let $K^{\prime \prime}$ be the set $K$ of Proposition 3.6 associated to $p^{\prime \prime}$; this is again an open and dense subset in the set of all $(q, \ell)$ in $T M$ such that $\ell$ is null, and such that the associated null geodesic in $M^{\prime \prime}$ ends at $p^{\prime \prime}$. Choosing any $(q, \ell)$ in $K^{\prime} \cap K^{\prime \prime}$, the corresponding null geodesic will have a transverse endpoint in both completions.

We introduce the following topology on $M_{\Xi}$ : Since, by hypothesis, $M$ is paracompact, there exist complete Riemannian metrics on $M$ [22]. Choose any such metric and let $d$ be the associated distance function. Similarly $T M$ is paracompact, and let $\hat{d}: T M \times T M \rightarrow \mathbb{R}^{+}$be the distance function associated to some complete Riemannian metric on $T M$. Again by paracompactness there exists a countable dense set $X_{i} \subset N M$, where $N M$ denotes the bundle of null vectors over $M$ (see, e.g., [22]). Consider the collection $\mathscr{B}$ consisting of the following sets: 
1) All $\hat{d}$-distance open balls $B_{i, j} \subset M$, of rational radii $\rho_{j}$, centred at the (countable, dense) collection of points $\pi\left(X_{i}\right)$, where $\pi$ is the projection map from $N M$ to $M$. (Unsurprisingly, the number $\rho_{j}$ will be called the radius of $B_{i, j}$.)

2) All thickenings $\mathscr{O}_{i, j}$ of null geodesics generated by those null vectors which belong to $\hat{d}$-distance balls of rational radii $r_{j}$ around those $X_{i}$ 's which acquire transverse endpoints in some extension $\hat{\alpha} \in \Xi$. Let $s_{i}$ be the affine time, in the conformally rescaled metric $\hat{g}$, taken by the geodesic generated by $X_{i}$ to reach the boundary $\partial \hat{M}$. The number $r_{i, j}=\max \left(s_{i}, r_{j}\right)$ will be called the radius of $\mathscr{O}_{i, j}$

The topology of $M_{\Xi}$ is defined as the topology generated by the equivalence classes of sets in $\mathscr{B}$ (the reader should easily check that the intersection condition of $[21$, p. 78$]$ is satisfied by $\mathscr{B})$. Thus, those equivalence classes provide a countable basis for the topology of $M_{\Xi}$. By an abuse of notation we will use the same symbol $\mathscr{B}$ for the collection of equivalence classes.

Now, we have charts on the set $M_{\Xi}$, which will give $M_{\Xi}$ the structure of a manifold with boundary provided $M_{\Xi}$ is a Hausdorff paracompact topological space. In order to establish the Hausdorff property, consider two distinct points $p_{a}, a=1,2$ in $M_{\Xi}$. We need to show that they can be separated by open neighborhoods. The only case that is not completely straightforward is $p_{a} \in M_{\Xi} \backslash M$. Let $p_{1}$ arise from a completion $\alpha^{\prime}$, and let $p_{2}$ arise from a completion $\alpha^{\prime \prime}$ (possibly equal to $\alpha^{\prime}$ ); since $p_{1} \neq p_{2}$ we are in case $i i$ ) of Definition 4.3, which implies that sufficiently small coordinate neighborhoods arising from coordinates near the respective boundaries around the $p_{a}$ 's will be disjoint.

In order to prove that $M_{\Xi}$ is a manifold, it remains to show that $M_{\Xi}$ is paracompact. We will show shortly that $M_{\Xi}$ is regular as a topological space. Since the topology of $M_{\Xi}$ has a countable basis, we can use a theorem of Urysohn [21, Theorem 4.1, p. 217] to conclude that the topology of $M_{\Xi}$ is metrisable. Paracompactness follows then from Smirnov's metrisation theorem [21, Theorem 5.1, p. 260].

So, we need to establish topological regularity. By definition [21, p. 195], we need to show that points in $M_{\Xi}$ are closed, and that for every $p \in M_{\Xi}$ and every closed set $C \subset M_{\Xi}$ not containing $p$ there exist disjoint open sets $\mathscr{O}$ and $\mathscr{U}$ with $p \in \mathscr{O}$ and $C \subset \mathscr{U}$. The fact that points are closed is a standard consequence of the Hausdorff property. On the other hand, the separation property follows from the existence of local charts, as follows: Let $p \in M_{\Xi}$, let $C$ be a closed subset of $M_{\Xi}$, for each $q \in M_{\Xi}$ let $U_{i(q)}$ be an element of the basis $\mathscr{B}$ containing $q$, and not containing $p$, with radius smaller than or equal to $1 / i$. Set

$$
U_{i}=\cup_{q \in C} U_{i(q)} .
$$


Similarly let $V_{i}$ be an element of $\mathscr{B}$ containing $p$ with radius smaller than or equal to $1 / i$. We wish to show that $V_{i} \cap U_{i}=\emptyset$ for $i$ large enough, and regularity will follow. Suppose that this last property does not hold; then there exists a sequence of points $q_{i} \in C$ such that $U_{i}\left(q_{i}\right) \cap V_{i} \neq \emptyset$. There exists $i_{0}$ such that for all $i \geq i_{0}$ all $V_{i}$ 's and $U_{i}\left(q_{i}\right)$ 's are contained in a single coordinate chart near $p$. Increasing $i_{0}$ further if necessary, the $V_{i}$ 's and the $U_{i}\left(q_{i}\right)$ 's are contained in coordinate balls with radii approaching zero as $i$ tends to infinity. Furthermore each $V_{i}$ contains some small coordinate ball centred at $p$. This shows that for every $j$ there exists $i$ large enough so that $U_{i}(q) \subset V_{j}$ if $U_{i}(q) \cap V_{i} \neq \emptyset$, which implies that $V_{j}$ intersects $C$, so that $p$ is in the closure of $C$, hence in $C$, a contradiction.

On $M_{\Xi}$ we construct a Lorentzian metric as follows: By paracompactness there exists a covering $\left\{\mathscr{O}_{i}\right\}_{i \in \Omega}$ of $M_{\Xi}$ by local coordinate charts, such that each chart is a local coordinate chart for some conformal boundary extension $\alpha_{i}=\left(M_{i}, g_{i}, \psi_{i}\right) \in \Xi$. Again by paracompactness there exists a partition of unity $\left\{\varphi_{i}\right\}_{i \in \mathbb{N}}$ subordinate to this covering. On $M$ define

$$
g_{\Xi}=\sum_{i \in \mathbb{N}} \varphi_{i} \psi_{i}^{*} g_{i}
$$

Note that a convex combination of Lorentzian metrics is not necessarily a Lorentzian metric; however the $\psi_{i}^{*} g_{i}$ 's are all conformal to each other, which makes it easy to check that $g_{\Xi}$ is indeed a Lorentzian metric on $M$, conformal to $g$.

Next, let $M \stackrel{\hat{\psi}_{\Xi}}{\rightrightarrows} M_{\Xi}$ be the map that sends $M$ to the " $M$ " (via the identity) in the disjoint union that is $M_{\Xi}$. The metric $g_{\Xi}$ extends smoothly to the boundary for any extension $\hat{\alpha}$, as a consequence of Theorem 1 , which guarantees that each tensor field $\left(\hat{\psi}^{-1}\right)^{*}\left(\varphi_{i} \psi_{i}^{*} g_{i}\right)$ smoothly extends from the interior of $\hat{M}$ to $\partial \hat{M}$. Using the same symbol $g_{\Xi}$ for the metric obtained by extending $g_{\Xi}$ to the boundary, this makes $\left(M_{\Xi}, g_{\Xi}, \hat{\psi}_{\Xi}\right)$ a conformal boundary extension. $\quad$ q.e.d.

\section{Applications}

All the results that follow are more or less straightforward consequences of Theorem 4.5. Let us start with the simplest application (compare [10]):

Theorem 5.1. Suppose that the collection of all future inextendible null geodesics of $(M, g)$ is simple, then there exists a unique, up to equivalence, maximal future conformal boundary extension of $(M, g)$.

REMARK 5.2. The maximal future conformal boundary extension will be empty if and only if there are no future conformal boundary extensions at all. 
Proof. We let $\Xi$ be the collection of all extensions of $(M, g)$, and apply Theorem 4.5.

q.e.d.

5.1. Strongly causal boundaries, Trautman-Bondi mass. Clearly, if a null geodesic $\gamma$ terminating at $p \in \partial \hat{M}$ is intertwined with some other null geodesic, then strong causality fails at $p$. Letting $\Xi$ be the collection of strongly causal future conformal boundary extensions, it should be clear that the future conformal boundary extension of Theorem 4.5 has a strongly causal boundary, so that we have:

Theorem 5.3. Every $(M, g)$ admits a unique, up to equivalence, completion which is maximal within the class of future conformal boundary extensions with strongly causal boundaries.

REMARK 5.4. The maximal future conformal boundary extension will be empty if and only if there are no future conformal boundary extensions of $(M, g)$ with strongly causal boundaries. Similar comments apply to our remaining existence results below, and will not be repeated.

As is well known, four-dimensional vacuum space-times with smooth conformal structure at null infinity can be constructed by solving a "hyperboloidal Cauchy problem" (see, e.g., [9] and references therein). This leads to non-empty strongly causal future conformal boundary extensions, and hence a unique maximal $\mathscr{I}$ by Theorem 5.3. This remains true in all higher even space-time dimensions [1].

One of the approaches to the definition of the Trautman-Bondi mass $[3,29]$ proceeds via Penrose's conformal framework [12]. This leads to a potential ambiguity, related to the possibility of existence of inequivalent differentiable structures of $\mathscr{I}$. Our analysis here shows that for strongly causal conformal completions at infinity no such ambiguities arise, which establishes well-posedness of the definitions in [12]. (Compare [6] for an alternative proof in dimension four.) The conformallyinvariant formula for the Trautman-Bondi mass of cuts of $\mathscr{I}$ in higher dimensions of Ashtekar and Das $[2,16]$ together with our result extends to all higher dimensions the four-dimensional statement here.

5.2. Spacelike conformal boundaries. Another straightforward application is that to existence and uniqueness of maximal spacelike boundaries:

Theorem 5.5. Every space-time $(M, g)$ admits a unique, up to equivalence, future conformal boundary extension which is maximal within the class of completions with spacelike boundary.

REMARK 5.6. The result does not, of course, exclude the possibility that there exist strictly larger future conformal boundary extensions $\alpha^{\prime \prime}$; however, any such $T \partial M^{\prime \prime}$ will contain non-spacelike tangent spaces. 
Proof. Let $\Omega_{\text {space }}$ denote the collection of all null geodesics which terminate at a point $p$ belonging to some conformal boundary extension $\hat{\alpha}$ such that $\partial \hat{M}$ is spacelike at $p$. Simplicity of $\Omega_{\text {space }}$ is a straightforward consequence of the next lemma, and the result follows then from Theorem 4.5 and Remark 4.6.

q.e.d.

Lemma 5.7. Suppose that $\gamma_{1}$ is intertwined with $\gamma_{2}$, with $\gamma_{1}$ terminating at $p \in \partial M^{\prime}$. Then $T_{p} \partial M^{\prime}$ contains a null direction.

Proof. By definition there exists a sequence $p_{i}=\psi^{\prime}\left(\gamma_{2}\left(s_{i}\right)\right)$ converging to $p$. Choosing a subsequence and changing the parameterisation if necessary, the sequence of null vectors $\psi_{*}^{\prime} \dot{\gamma}_{2}\left(s_{i}\right)$ converges then to a null vector tangent to $\partial M^{\prime}$ at $p$.

q.e.d.

5.3. Null conformal boundaries. An important class of conformal boundary extensions is provided by those for which the boundary is a null manifold. We note the following corollary of Lemma 5.7:

Proposition 5.8. Let $\alpha^{\prime}$ be a completion of $(M, g)$ with $\partial M^{\prime}$ null, and let $\gamma_{1}$ terminate at $p \in \partial M^{\prime}$. Then $\gamma_{2}$ intertwines $\gamma_{1}$ if and only if $\gamma_{2}$ accumulates at the null generator of $\partial M^{\prime}$ through $p$.

Proof. Let $\gamma$ be the null geodesic generator of $\partial M$ so that $\gamma(0)=p$. We identify $\gamma_{a}$ with $\psi^{\prime}\left(\gamma_{a}\right)$. In order to prove the implication " $\Rightarrow$," note that the proof of Lemma 5.7 shows that there exists a parameterisation of $\gamma_{2}$ so that the sequence $\left(\gamma_{2}\left(s_{i}\right), \dot{\gamma}_{2}\left(s_{i}\right)\right)$ approaches $(\gamma(0), \dot{\gamma}(0))$ as $i$ tends to infinity, and the result follows from continuous dependence of geodesics upon initial values. To prove the reverse implication, consider any neighborhood $\mathscr{O}$ of $p$ such that $\left(\mathscr{O},\left.g\right|_{\mathscr{O}}\right)$ is strongly causal. Let $s_{i}$ be an increasing parameter sequence such that $\left(\gamma_{2}\left(s_{i}\right), \dot{\gamma}_{2}\left(s_{i}\right)\right)$ approaches $(\gamma(0), \dot{\gamma}(0))$ as $i$ tends to infinity. Suppose that $\gamma_{2}$ is entirely contained in $\mathscr{O}$. Standard causality theory shows that $\gamma_{2}$ can then be extended by the generator of $\mathscr{O}$ at $\gamma(0)$ and hence coincides with this generator near $p$, which is not possible. It follows that $\gamma_{2}$ leaves and reenters all sufficiently small neighborhoods of $p$ an infinite number of times, and consequently intertwines $\gamma_{1}$.

q.e.d.

5.4. Simple space-times. Theorem 5.1 can be sharpened somewhat: Let $\Omega_{\mathrm{fcb}}$ be the collection of all future inextendible null geodesics of $(M, g)$ satisfying the following property: $\gamma \in \Omega_{\mathrm{fcb}}$ if and only if there exists some future conformal boundary extension $\alpha_{\gamma}$ of $(M, g)$ which terminates $\gamma$. (The subscript "fcb" stands for "future conformal boundary.") This is clearly an open collection of geodesics. Applying Theorem 4.5 to the collection

$$
\Xi_{\mathrm{fcb}}=\left\{\alpha_{\gamma}, \gamma \in \Omega_{\mathrm{fcb}}\right\}
$$

of all conformal boundary extensions we obtain: 
Theorem 5.9. There exists a unique, up to equivalence, maximal future conformal boundary extension $(\hat{M}, \hat{g})$ of $(M, g)$ if and only if $\Omega_{\mathrm{fcb}}$ is simple. In particular, if that last condition holds, then every future conformal boundary extension of $(M, g)$ is equivalent to a subset of $(\hat{M}, \hat{g})$.

We shall say that a manifold $(M, g)$ is simple to the future if $(M, g)$ is conformal to the interior of a manifold $\left(M_{s}, g_{s}\right)$ with smooth boundary, with each future inextendible null geodesic in $(M, g)$ acquiring an endpoint on the boundary of $M_{s}$. This is an obvious extension of a similar definition of Penrose [23]. Theorem 5.9 gives:

Theorem 5.10. Let $(M, g)$ be simple to the future. Then $\left(M_{s}, g_{s}\right)$ is the maximal conformal future boundary extension of $(M, g)$, and hence unique up to equivalence.

5.5. Counting maximal extensions. Let $\mathscr{N}_{\text {term }}$ denote the collection of simple sets $\Omega$ which are maximal in the class that contains only those geodesics which are terminated by some conformal boundary extension of $M$. Theorem 5.9 shows that every such nonempty $\Omega$ defines a unique maximal conformal boundary extension. We thus have:

Theorem 5.11. The set of inequivalent maximal future conformal boundary extensions of $(M, g)$ is in one-to-one correspondence with $\mathscr{N}_{\text {term }}$.

Since any $\Omega(\hat{\alpha})$, as defined in Proposition 4.4 , can be completed to a maximal simple $\Omega$, the number of maximal future conformal boundary extensions of $(M, g)$ is smaller than or equal to the number of simple maximal collections of future inextendible geodesics in $M$. Thus, Theorem 5.11 gives an upper bound on the number of extensions, without any knowledge about their existence.

5.6. Cauchy horizons. An important class of conformal boundary extensions is provided by Cauchy horizons. In that case the boundary is necessarily a null topological hypersurface. We start with the following:

Proposition 5.12. Consider a globally hyperbolic space-time $(M, g)$, and let $\hat{\alpha}$ be a future conformal boundary extension such that $\partial \hat{M}$ is null and the following holds: Every future inextendible null geodesic $\gamma$ in $M$ accumulates at $\partial \hat{M}$. Then $\Omega(\hat{\alpha})$ is maximal.

Proof. By hypothesis there exists a sequence $p_{i}=\hat{\psi}\left(\gamma\left(s_{i}\right)\right)$ such that $p_{i} \rightarrow p$ for some $p \in \partial \hat{M}$. Reparameterizing and passing to a subsequence if necessary the sequence $\hat{\psi}_{*} \dot{\gamma}\left(s_{i}\right)$ converges to a null vector $\ell$ at $p$. If $\ell$ is transverse to $\partial \hat{M}$, then $p$ is a future endpoint of $\hat{\psi}(\gamma)$ and thus $\gamma \in \Omega(\hat{\alpha})$. If not, then $\ell$ is tangent to the generator of $\partial \hat{M}$ through $p$, and $\gamma \notin \Omega(\hat{\alpha})$ follows from Proposition 5.8. $\quad$ q.e.d. 
We note:

Proposition 5.13. Consider a globally hyperbolic space-time $(M, g)$, and let $\hat{\alpha}$ be a future conformal boundary extension with $\partial \hat{M}$ null and compact. Then the hypotheses (and hence the conclusions) of Proposition 5.12 hold.

Proof. It is well known that $\hat{M}$ is diffeomorphic to

$$
(-1,1] \times \partial \hat{M}
$$

with every level set $\{s\} \times \partial \hat{M}$ being a spacelike Cauchy surface for $\hat{M} \backslash \partial \hat{M}$. If we denote by $t$ the projection along the first factor in (7), then $t \circ \psi$ approaches one along any future inextendible causal geodesic in $M$, and compactness of $\partial \hat{M}$ implies that $\psi(\gamma)$ accumulates at some $p \in \partial \hat{M}$.

q.e.d.

5.7. Taub-NUT space-time. The globally hyperbolic region of TaubNUT space-time $(M, g)$ has two known inequivalent (but isometric) future conformal boundary extensions $[5,13,19]$, say $\alpha_{1}$ and $\alpha_{2}$, with boundaries $S^{3}$. We will show that subsets of $\alpha_{1}$ can be used to classify all extensions. To fix notation, we parameterise the Taub region of the Taub-NUT space-time with $t \in\left(t_{-}, t_{+}\right)$and with Euler angles $(\zeta, \theta, \varphi)$ on $S^{3}$, with the metric taking the form

$$
g=-U^{-1} d t^{2}+(2 \ell)^{2} U(d \zeta+\cos \theta d \varphi)^{2}+\left(t^{2}+\ell^{2}\right)\left(d \theta^{2}+\sin ^{2} \theta d \varphi^{2}\right),
$$

where

$$
\begin{aligned}
U(t) & =\frac{\left(t_{+}-t\right)\left(t-t_{-}\right)}{t^{2}+\ell^{2}}, \\
t_{ \pm} & :=m \pm \sqrt{m^{2}+\ell^{2}} .
\end{aligned}
$$

Here $\ell$ and $m$ are strictly positive constants.

The future extension $\alpha_{1}$ is obtained as follows: One sets $M_{1}=$ $\left(t_{-}, t_{+}\right] \times S^{3}$, with metric

$$
\begin{aligned}
g_{1}=-4 \ell(d \zeta & +\cos \theta d \varphi) d t+(2 \ell)^{2} U(d \zeta+\cos \theta d \varphi)^{2} \\
& +\left(t^{2}+\ell^{2}\right)\left(d \theta^{2}+\sin ^{2} \theta d \varphi^{2}\right),
\end{aligned}
$$

with the map $\psi_{1}$ begin given by the formula

$$
(t, \zeta, \theta, \varphi) \mapsto\left(t, \zeta-(2 \ell)^{-1} \int_{0}^{t} U^{-1}(x) d x, \theta, \varphi\right) .
$$

With this definition one has $\left(\psi_{1}\right)^{*} g_{1}=g$. The extension $\alpha_{2}$ is obtained by changing two signs above: thus $M_{2}=\left(t_{-}, t_{+}\right] \times S^{3}$, with metric

$$
\begin{aligned}
g_{2}=4 \ell(d \zeta & +\cos \theta d \varphi) d t+(2 \ell)^{2} U(d \zeta+\cos \theta d \varphi)^{2} \\
& +\left(t^{2}+\ell^{2}\right)\left(d \theta^{2}+\sin ^{2} \theta d \varphi^{2}\right),
\end{aligned}
$$


with the map $\psi_{2}$ being given by

$$
(t, \zeta, \theta, \varphi) \mapsto\left(t, \zeta+(2 \ell)^{-1} \int_{0}^{t} U^{-1}(x) d x, \theta, \varphi\right),
$$

leading similarly to $\left(\psi_{2}\right)^{*} g_{2}=g$. Note that the transformation $(\zeta, \varphi) \rightarrow$ $(-\zeta,-\varphi)$ transforms $g_{1}$ into $g_{2}$, so that $\left(M_{1}, g_{1}\right)$ and $\left(M_{2}, g_{2}\right)$ are isometric.

The vector field $\partial_{\zeta}$ is clearly a Killing vector for all three metrics above, and therefore

$$
p_{\|}:=g\left(\partial_{\zeta}, \dot{\gamma}\right)
$$

is constant along the geodesic $\gamma$. Taub space-time has three further Killing vectors, the exact form of which is irrelevant to us here; they lead to constants of motion which are denoted by $p_{a}, a=1,2,3$. It is proved in [13] (see Theorems 1-3 and the Appendix there) that:

1) every inextendible future directed null geodesic with $p_{\|}>0$ acquires an endpoint on $\partial M_{1}$;

2) similarly every inextendible future directed null geodesic with $p_{\|}<$ 0 is terminated by $\partial M_{2}$;

3) finally, for every inextendible future directed null geodesic $\gamma(s)$ : $\left[0, s_{+}\right) \rightarrow M$ with $p_{\|}=0$ the functions $\zeta(s), \theta(s)$, and $\varphi(s)$ have finite limits as $s$ tends to the end value $s_{+}$.

We will need the following:

Lemma 5.14. There exist no extension of the Taub space-time $(M, g)$ which terminates null geodesics with $p_{\|}=0$.

Proof. We will argue by contradiction: Let $\gamma:\left[0, s_{+}\right) \rightarrow M$ satisfy $p_{\|}=0$ and suppose that there exists an extension $\hat{\alpha}$ terminating $\gamma$. Then there exists a neighborhood $\mathscr{O}$ of $\dot{\gamma}(0)$ in $\mathscr{N} M$ such that no null geodesics with initial values in $\mathscr{O}$ are intertwined with $\gamma$. We will show that no such neighborhood exists, establishing the result.

We thus need to prove that any neighbourhood $\mathscr{O}$ of $\dot{\gamma}(0)$ contains a null geodesic $\hat{\gamma}$ which is intertwined with $\gamma$. We seek to show that an appropriate geodesic with $\hat{p}_{\|}=\epsilon \neq 0, \epsilon$ small, will have that property.

It follows from the equations in [20] that the angular coordinates can always be chosen so that $\sin \theta$ is strictly bounded away from zero on any given geodesic and on nearby ones. Recall, further, the relation [20]

$$
\sin \theta \cos \varphi p_{1}+\sin \theta \sin \varphi p_{2}+\cos \theta p_{3}=p_{\|} \cdot
$$

Clearly for any $\epsilon$ we can choose initial data for $\hat{\gamma}$, near to those for $\gamma$, so that $\hat{p}_{i} \rightarrow p_{i}$ as $\epsilon \rightarrow 0$.

Let $\theta_{\infty}$ and $\varphi_{\infty}$ be the limiting values of $\theta(s)$ and $\varphi(s)$ as $s$ approaches the terminal value $s_{+}$(those exist from what has been said above; in any case this will be clear from what follows). We want to show that $\hat{\gamma}(0)$ 
can be chosen so that the corresponding values $\hat{\theta}_{\infty}$ and $\hat{\varphi}_{\infty}$ for $\hat{\gamma}$ coincide with those for $\gamma$. The coordinates $\theta$ and $\varphi$ satisfy the equations [20]

$$
\frac{d \varphi(s(t))}{d t}=\frac{\hat{p}_{3}}{\left(t^{2}+\ell^{2}\right) \sin ^{2} \theta \sqrt{\left(\frac{p_{\|}}{2 \ell}\right)^{2}+U\left(\frac{\left(p_{2} \cos \varphi-p_{1} \sin \varphi\right)^{2}}{t^{2}+\ell^{2}}+\frac{p_{3}^{2}}{\left(t^{2}+\ell^{2}\right) \sin ^{2} \theta}\right)}}
$$

$$
\frac{d \theta(s(t))}{d t}=\frac{p_{2} \cos \varphi-p_{1} \sin \varphi}{\left(t^{2}+\ell^{2}\right) \sqrt{\left(\frac{p_{\|}}{2 \ell}\right)^{2}+U\left(\frac{\left(p_{2} \cos \varphi-p_{1} \sin \varphi\right)^{2}}{t^{2}+\ell^{2}}+\frac{p_{3}^{2}}{\left(t^{2}+\ell^{2}\right) \sin ^{2} \theta}\right)}},
$$

along $\hat{\gamma}$, with the corresponding equations for $\gamma$ obtained by setting $p_{\|}=0$. We need to make sure that the value $\hat{\varphi}_{\infty}-\varphi_{\infty}$ approaches zero as $p_{\|}=\epsilon \rightarrow 0$; note that the behaviors of the right-hand sides in (14)-(15) are drastically different depending upon whether or not $p_{\|}=0$ because $U$ tends to zero as $t \rightarrow t_{+}$. However, for any $t \neq t_{+}$ the right-hand sides of (14)-(15) converge to the ones with $p_{\|}=0$ as $p_{\|}$ approaches zero. Further we have the obvious estimate

$$
\left|\frac{d \varphi(s(t))}{d t}\right| \leq \frac{1}{\sin \theta \sqrt{\left(t^{2}+\ell^{2}\right) U}}=\frac{1}{\sin \theta \sqrt{\left(t_{+}-t\right)\left(t-t_{-}\right)}} .
$$

Similarly

$$
\left|\frac{d \theta(s(t))}{d t}\right| \leq \frac{1}{\sqrt{\left(t_{+}-t\right)\left(t-t_{-}\right)}}
$$

The functions at the right-hand sides of (16)-(17) belong to $L^{1}\left(\left[t_{-}, t_{+}\right]\right)$, and the conclusion that

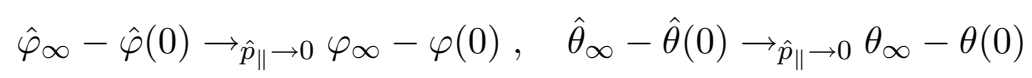

follows from Lebesgue's dominated convergence theorem.

Standard considerations based on the Brouwer fixed-point theorem show that initial values $(\hat{\varphi}(0), \hat{\theta}(0))$ can be found so that

$$
\hat{\varphi}_{\infty}=\varphi_{\infty}, \quad \hat{\theta}_{\infty}=\theta_{\infty},
$$

with $(\hat{\varphi}(0), \hat{\theta}(0))$ being as close to $(\varphi(0), \theta(0))$ as desired if $\hat{p}_{\|}$is chosen sufficiently small. In conclusion, a sufficiently small $\hat{p}_{\|}$will lead to a $\hat{\gamma}(0)$ belonging to $\mathscr{O}$, for any neighbourhood $\mathscr{O}$ of $\gamma(0)$.

It is convenient to parameterise both $\gamma$ and $\hat{\gamma}$ by $t$. Since $\hat{p}_{\|}>0$ the geodesic $\hat{\gamma}$ has an endpoint on $\partial M_{1}$, we thus have

$$
\left(\hat{\zeta}_{1}(t), \hat{\theta}(t), \hat{\varphi}(t)\right) \rightarrow_{t \rightarrow \hat{t}_{+}}\left(\hat{\alpha}, \theta_{\infty}, \varphi_{+}\right),
$$

for some constant $\hat{\alpha} \in \mathbb{R}$, where

$$
\hat{\zeta}_{1}(t)=\hat{\zeta}(t)-(2 \ell)^{-1} \int_{0}^{t} U^{-1}(x) d x
$$


We define $\zeta_{1}(t)$ by an obvious equivalent of (19). On the other hand

$$
(\zeta(t), \theta(t), \varphi(t)) \rightarrow_{t \rightarrow t_{+}}\left(\zeta_{+}, \theta_{\infty}, \varphi_{+}\right) .
$$

The integral appearing in (19) diverges logarithmically as $t \rightarrow t_{+}$, leading to

$$
\zeta_{1}(t) \rightarrow_{t \rightarrow t_{+}}-\infty
$$

Summarising,

$\theta(t)-\hat{\theta}(t) \rightarrow_{t \rightarrow t_{+}} 0, \quad \varphi(t)-\hat{\varphi}(t) \rightarrow_{t \rightarrow t_{+}} 0, \quad \zeta_{1}(t)-\hat{\zeta}_{1}(t) \rightarrow_{t \rightarrow t_{+}}-\infty$.

Consider, now, any small thickening of $\hat{\gamma}$ contained in a set $\mathscr{U}$ of the form

$\left\{t \in\left(t_{+}-\delta, t_{+}\right), \zeta_{1} \in(\hat{\alpha}-\delta, \hat{\alpha}+\delta), \theta \in\left(\theta_{\infty}-\delta, \theta_{\infty}+\delta\right), \varphi \in\left(\varphi_{+}-\delta, \varphi_{+}+\delta\right)\right\}$,

for some small $\delta$. Since $\zeta$ and $\zeta_{1}$ are periodic coordinates on $S^{3}$, both $\zeta$ and $\zeta_{1}$ are identified modulo $2 \pi$. This implies that $\gamma$ will visit and leave $\mathscr{U}$ an infinite number of times, no matter how small $\delta$ is, showing that $\gamma$ and $\hat{\gamma}$ are intertwined, as desired.

q.e.d.

The reader will note that a simple variation of the arguments just given proves the following:

Proposition 5.15. Two future inextendible geodesics $\gamma$ and $\hat{\gamma}$ in Taub space-time are intertwined if and only if

$$
\lim _{t \rightarrow t_{+}} \varphi(t)-\hat{\varphi}(t)=\lim _{t \rightarrow t_{+}} \theta(t)-\hat{\theta}(t)=0, \quad p_{\|} \hat{p}_{\|} \leq 0, p_{\|} \neq \hat{p}_{\|} .
$$

The following provides a classification of boundary extensions of the Taub region:

Proposition 5.16. Any future conformal boundary extension $\hat{\alpha}$ of Taub space-time, or of a Gowdy space-time, with a connected boundary is equivalent to a subset of $\alpha_{1}$ or of $\alpha_{2}$. In particular $\hat{\alpha}$ is equivalent to $\alpha_{1}$ or $\alpha_{2}$ when $\partial \hat{M}$ is compact.

Before passing to its proof, note that Proposition 5.16 also follows from [8, Theorem 5.2] when the boundary is compact, because for compact boundaries the action of the connected component of the group of isometries extends to the boundary. The proof in that last reference appears to be completely different, making heavy use of the structure of the isometry group of the space-time. It should, however, be noted that the large group of isometries is also used in our proof here, in analysing the geometry of the set of geodesics.

Proof. Consider any null geodesic $\gamma$ terminated transversally by $\partial \hat{M}$. Lemma 5.14 shows that $\gamma$ has a future endpoint either on $\alpha_{1}$ or on $\alpha_{2}$. Let $\mathscr{U}_{a} \subset \partial \hat{M}$ denote the set of endpoints of null geodesics which also terminate on $\partial M_{a}$. Then each $\mathscr{U}_{a}$ is open, at least one of them is not 
empty, renaming the $\alpha_{a}$ 's if necessary it follows that $\mathscr{U}_{1}=\partial \hat{M}$. This implies that no geodesics terminated by $\partial \hat{M}$ and $\partial M_{1}$ are intertwined, and we can apply Theorem 4.5 with $\Xi=\left\{\alpha_{1}, \hat{\alpha}\right\}$ to obtain a new boundary $M_{\Xi}$ in which both $M_{1}$ and $\hat{M}$ are embedded. Proposition 5.13 implies that $\alpha_{\Xi}$ is equivalent to $\alpha_{1}$, and the result follows.

q.e.d.

We finish this section by providing a large family of examples of maximal simple $\Omega$ 's. We start with the polarized Gowdy case; a similar construction applies in Taub space-times. The Gowdy space-times have $\mathbb{R} \times S^{1} \times S^{1} \times S^{1}$ topology, with the last two factors being acted upon by $U(1) \times U(1)$ by isometries in the obvious way (see e.g., [5]). If we denote by $(t, \theta)$ the coordinates on the first $\mathbb{R} \times S^{1}$ factor, and by $\left(x^{a}\right)$ the coordinates on the remaining factors, then (after a convenient choice of the $x^{a}$ 's) for every $x=\left(\theta, x^{1}\right) \in S^{1} \times S^{1}$ the geodesic $\gamma_{L}(x)$ lying in the $\left(t, x^{2}\right)$ plane going to, say, the left, has an endpoint in $\partial M_{1}$ and intertwines the remaining geodesic in that plane $\gamma_{R}(x)$, which has an endpoint on $\partial M_{2}$. Choose any non-empty open set $\mathscr{U}_{1} \subset \mathbb{T}^{2}=$ $S^{1} \times S^{1}$, with the $S^{1}$ 's here corresponding to the $\theta^{\text {'s }}$ and $x^{1}$ 's, such that the interior of the closure $\overline{\mathscr{U}}_{1}$ of $\mathscr{U}_{1}$ coincides with $\mathscr{U}_{1}$, and let $\mathscr{U}_{2}=\mathbb{T}^{2} \backslash \overline{\mathscr{U}}_{1}$. (Equivalently, $\mathscr{U}_{1}$ and $\mathscr{U}_{2}$ are disjoint open subsets of $\mathbb{T}^{2}$ such that $\partial \mathscr{U}_{1}=\partial \mathscr{U}_{2}$ and $\overline{\mathscr{U}}_{1} \cup \overline{\mathscr{U}}_{2}=\mathbb{T}^{2}$.) Let $\Omega_{\mathscr{U}_{1}}$ be the union of the collection of sufficiently small neighborhoods (in the space of inextendible geodesics) of left-going $\gamma_{L}(x)$ as $x$ runs over $\mathscr{U}_{1}$ with the collection of small neighborhoods of the right-going $\gamma_{R}(x)$ as $x$ runs over $\mathscr{U}_{2}$. It should be clear that the neighborhoods can be so chosen that $\Omega_{\mathscr{U}_{1}}$ is simple, and that any maximal simple $\hat{\Omega}_{\mathscr{U}_{1}}$ containing $\Omega_{\mathscr{U}_{1}}$ will satisfy $\hat{\Omega}_{\mathscr{U}_{1}} \neq \hat{\Omega}_{\mathscr{U}_{1}^{\prime}}$ if $\mathscr{U}_{1} \neq \mathscr{U}_{1}^{\prime}$.

An obvious adaptation of the discussion of the last paragraph to the Taub space-time, together with Proposition 5.16, shows that all maximal future boundary extensions of the Taub space-time are in oneto-one correspondence with open non-empty sets $\mathscr{U}_{1} \subset S^{2}$, where $S^{2}$ here corresponds to the Hopf quotient of $\partial M_{1} \approx S^{3}$, such that the interior of the closure $\overline{\mathscr{U}}_{1}$ of $\mathscr{U}_{1}$ coincides with $\mathscr{U}_{1}$. If $\pi: S^{2} \rightarrow S^{2}$ denotes the Hopf projection, then $\pi^{-1} \mathscr{U}_{1} \subset \partial M_{1}$ is the set of endpoints of null geodesics terminated by a subset of $\partial M_{1}$, while $\pi^{-1} \mathscr{U}_{2} \subset \partial M_{2}$ is the corresponding subset of $\partial M_{2}$.

\section{6. "Minimally non-Hausdorff" maximal extensions?}

Following [14], a topological space will be called a $Y$-manifold if every point has a neighborhood homeomorphic to an open subset of $\mathbb{R}^{n}$, with the charts satisfying the usual smooth compatibility conditions. Thus, one does not require a $Y$-manifold to be either Hausdorff or paracompact. An example can be provided by attaching simultaneously $\partial M_{1}$ and $\partial M_{2}$ to the Taub space, with the obvious charts near the boundaries. 
It should be clear from what has been said above that every conformal boundary extension of Taub-NUT space with conformal factor one can be viewed as a subset of this extension.

In general, let $\Xi$ be the collection of all future conformal boundary extensions of $(M, g)$. Then the $Y$-manifold $M_{\Xi}$ constructed in the proof of Theorem 4.5 provides a $Y$-manifold which resembles this last nonHausdorff boundary extension of Taub space. (The reader will have noticed that the condition of simplicity of $\cup_{\alpha^{\prime} \in \Xi} \Omega\left(\alpha^{\prime}\right)$ in Theorem 4.5 has only been used to show that $M_{\Xi}$ is Hausdorff.)

To make this construction useful a better understanding of $M_{\Xi}$ would be necessary: is $M_{\Xi}$ paracompact? Does it carry a conformal structure? (Note that in Theorem 4.5 we used the former to construct the latter.) We have not attempted to analyse those questions. However, if one is only interested in Lorentzian extensions, i.e., conformal boundary extensions with conformal factor one - this is the case when considering e.g., Cauchy horizons - then the existence of a Lorentzian metric on $M_{\Xi}$ is immediate.

Assuming that $M_{\Xi}$ can be equipped with a conformal structure, it is then larger than or equal to any manifold conformal boundary extension of $(M, g)$ by construction. Moreover, again by construction, it is minimal with respect to this property. The sense in which this renders $M_{\Xi}$ unique needs to be made precise yet.

\section{References}

[1] M.T. Anderson \& P.T. Chruściel, Asymptotically simple solutions of the vacuum Einstein equations in even dimensions, Commun. Math. Phys. 260 (2005), 557577, arXiv:gr-qc/0412020. MR 2183957, Zbl 1094.83002.

[2] A. Ashtekar \& S. Das, Asymptotically anti-de Sitter space-times: Conserved quantities, Class. Quantum Grav. 17 (2000), L17-L30, arXiv:hep-th/9911230. MR 1739432, Zbl 0943.83023.

[3] H. Bondi, M.G.J. van der Burg \& A.W.K. Metzner, Gravitational waves in general relativity VII: Waves from axi-symmetric isolated systems, Proc. Roy. Soc. London A 269 (1962), 21-52. MR 0147276, Zbl 0106.41903.

[4] P.T. Chruściel \& M. Herzlich, The mass of asymptotically hyperbolic Riemannian manifolds, Pacific J. Math. 212 (2003), 231-264, arXiv:dg-ga/0110035. MR 2038048, Zbl 1056.53025.

[5] P.T. Chruściel \& J. Isenberg, Non-isometric vacuum extensions of vacuum maximal globally hyperbolic space-times, Phys. Rev. D (3) 48 (1993), 1616-1628. MR 1236815

[6] P.T. Chruściel, J. Jezierski \& S. Łȩski, The Trautman-Bondi mass of hyperboloidal initial data sets, Adv. Theor. Math. Phys. 8 (2004), 83-139, arXiv:grqc/0307109. MR 2086675, Zbl 1086.81066.

[7] P.T. Chruściel, M.A.H. MacCallum \& D. Singleton, Gravitational waves in general relativity. XIV: Bondi expansions and the "polyhomogeneity" of Scri, Philos. Trans. Roy. Soc. London Ser. A 350 (1995), 113-141. MR 1325206, Zbl 0829.53065 . 
[8] P.T. Chruściel \& A. Rendall, Strong cosmic censorship in vacuum space-times with compact, locally homogeneous Cauchy surfaces, Ann. Physics 242 (1995), 349-385. MR 1349391, Zbl 0831.53068.

[9] H. Friedrich, Einstein's equation and geometric asymptotics, Gravitation and Relativity: At the turn of the Millenium (Pune) (N. Dadhich and J. Narlikar, eds.), IUCAA, 1998, Proceedings of GR15, pp. 153-176.

[10] R. Geroch, Asymptotic structure of space-time, Asymptotic structure of spacetime (Proc. Sympos., Univ. Cincinnati, Cincinnati, Ohio, 1976), Plenum, New York, 1977, pp. 1-105. MR 0484240

[11] R. Geroch, E.H. Kronheimer \& R. Penrose, Ideal points in space-time., Proc. Roy. Soc. London Ser. A 327 (1972), 545-567. MR 0316035, Zbl 0257.53059.

[12] R. Geroch \& J. Winicour, Linkages in general relativity, Jour. Math. Phys. 22 (1981), 803-812. MR 617326, Zbl 0479.70004.

[13] P. Hájíček, Extensions of the Taub and NUT spaces and extensions of their tangent bundles, Commun. Math. Phys. 17 (1970), 109-126. MR 0264958, Zbl 0192.59302 .

[14] - Causality in non-Hausdorff space-times, Commun. Math. Phys. 21 (1971), 75-84. MR 0280143, Zbl 0215.23503.

[15] S.G. Harris, Boundaries on space-times: an outline, Advances in differential geometry and general relativity, Contemp. Math., vol. 359, Amer. Math. Soc., Providence, RI, 2004, arXiv:gr-qc/0310075, pp. 65-85. MR 2096154, Zbl 1083.53065 .

[16] S. Hollands, A. Ishibashi \& D. Marolf, Comparison between various notions of conserved charges in asymptotically AdS space-times, Class. Quantum Grav. 22 (2005), 2881-2920, arXiv:hep-th/0503045. MR 2154192, Zbl 1082.83014.

[17] J.A. Valiente Kroon, Nonexistence of conformally flat slices in Kerr and other stationary space-times, Phys. Rev. Lett. 92 (2004), 041101, 4 pp., arXiv:grqc/0310048. MR 2067779

[18] M.D. Kruskal, Maximal extension of Schwarzschild metric, Phys. Rev. (2) 119 (1960), 1743-1745. MR 0115757, Zbl 0098.19001.

[19] C.W. Misner, Taub-NUT space as a counterexample to almost anything, Relativity Theory and Astrophysics, AMS, Providence, Rhode Island, 1967, Lectures in Appl. Math., vol. 8, pp. 160-169.

[20] C.W. Misner \& A. Taub, A singularity-free empty universe, Soviet. Phys. JEPT 28 (1969), 122-133.

[21] J.R. Munkres, Topology: a first course, Prentice-Hall Inc., Englewood Cliffs, N.J., 1975. MR 0464128, Zbl 0306.54001.

[22] K. Nomizu \& H. Ozeki, The existence of complete Riemannian metrics, Proc. Amer. Math. Soc. 12 (1961), 889-891. MR 0133785, Zbl 0102.16401.

[23] R. Penrose, Zero rest-mass fields including gravitation: Asymptotic behaviour, Proc. Roy. Soc. London Ser. A 284 (1965), 159-203. MR 0175590, Zbl 0129.41202 .

[24] B.G. Schmidt, A new definition of conformal and projective infinity of spacetimes, Commun. Math. Phys. 36 (1974), 73-90. MR 0339775, Zbl 0282.53042.

[25] - On the uniqueness of boundaries at infinity of asymptotically flat spacetimes, Class. Quantum Grav. 8 (1991), 1491-1504. MR 1122247 
[26] - Errata: "On the uniqueness of boundaries at infinity of asymptotically flat spacetimes", Class. Quantum Grav. 9 (1992), 317-318. MR 1146065

[27] S.M. Scott \& P. Szekeres, The Curzon singularity. II: Global picture, Gen. Rel. Grav. 18 (1986), 571-583. MR 846735, Zbl 0588.53061.

[28] Gy. Szekeres, On the singularities of a Riemannian manifold, Gen. Rel. Grav. 34 (2002), 2001-2016, Reprinted from Publ. Math. Debrecen 7 (1960), 285-301 [ MR 0125541]. MR 1945497, Zbl 1049.83022.

[29] A. Trautman, King College Lectures on general relativity, May-June 1958, Gen. Rel. Grav. 34 (2002), 715-762. MR 1909313, Zbl 1004.83001.

FÉdÉration Denis Poisson, LMPT, Tours, And Albert Einstein Institute, Golm

E-mail address: Piotr.Chrusciel@lmpt.univ-tours.fr 